International Journal of Pure and Applied Mathematics

Volume 109 No. $4 \quad 2016,959-974$

ISSN: 1311-8080 (printed version); ISSN: 1314-3395 (on-line version)

url: http://www.ijpam.eu

doi: 10.12732/ijpam.v109i4.17

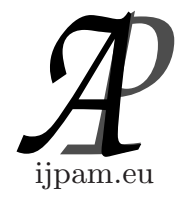

\title{
EXPLICIT MOORE-PENROSE INVERSE AND GROUP INVERSE OF DOUBLY LESLIE MATRIX
}

\author{
Wiwat Wanicharpichat \\ Department of Mathematics \\ Faculty of Science \\ Phitsanulok 65000, THAILAND \\ and \\ Research Center for Academic Excellence in Mathematics \\ Naresuan University \\ Phitsanulok 65000, THAILAND
}

\begin{abstract}
A doubly Leslie matrix is a bordered real matrix of the form

$$
L=\left[\begin{array}{cc}
-\mathbf{p}^{T} & -a_{n}-b_{n} \\
\Lambda & -\mathbf{q}
\end{array}\right]_{(n, n)},
$$

where $a_{n}, b_{n} \in \mathbb{R}, \mathbf{p}, \mathbf{q} \in \mathbb{R}^{n-1}$, and $\Lambda=\operatorname{diag}\left(s_{1}, s_{2}, \ldots, s_{n-1}\right)$ is a diagonal matrix of order $n-1$. The matrix $L$ is a closed form of a doubly companion matrix, a Leslie matrix and a companion matrix. This paper is discussed the explicit formula of the Moore-Penrose inverse and the group inverse of the doubly leslie matrix. In general the Moore-Penrose inverse of a rectangle doubly Leslie matrix is also discussed.
\end{abstract}

AMS Subject Classification: 15A09, 15A23

Key Words: companion matrix, doubly companion matrix, Leslie matrix, doubly Leslie matrix, Moore-Penrose inverse, group inverse

\section{Introduction}

One of the most popular models of population growth is a matrix-based model, first introduced by P.H. Leslie. In 1945, he published his most famous article in Biometrika, a journal. The article was entitled, On the use of matrices in

Received:

Revised:

Published:
August 19, 2016

September 14, 2016

October 8, 2016
(C) 2016 Academic Publications, Ltd. url: www.acadpubl.eu 
certain population mathematics [2, pp. 117-120]. The Leslie model describes the growth of the female portion of a population which is assumed to have a maximum lifespan. The females are divided into age classes all of which span an equal number of years. Using data about the average birthrates and survival probabilities of each class, the model is then able to determine the growth of the population over time, [6].

A Leslie matrix arises in a discrete, age-dependent model for population growth. It is a matrix of the form

$$
\mathbf{L}=\left[\begin{array}{cccccc}
r_{1} & r_{2} & r_{3} & \ldots & r_{n-1} & r_{n} \\
s_{1} & 0 & 0 & \ldots & 0 & 0 \\
0 & s_{2} & 0 & \ldots & 0 & 0 \\
\vdots & \vdots & \ddots & & \vdots & \vdots \\
0 & 0 & 0 & \ddots & 0 & 0 \\
0 & 0 & 0 & \ldots & s_{n-1} & 0
\end{array}\right]
$$

where $r_{j} \geq 0,0<s_{j} \leq 1, j=1,2, \ldots, n-1$.

Doubly companion matrices $C \in M_{n}$ were first introduced by Butcher and Chartier in [4, pp.274-276], given by

$$
C=\left[\begin{array}{cccccc}
-\alpha_{1} & -\alpha_{2} & -\alpha_{3} & \ldots & -\alpha_{n-1} & -\alpha_{n}-\beta_{n} \\
1 & 0 & 0 & \ldots & 0 & -\beta_{n-1} \\
0 & 1 & 0 & \ldots & 0 & -\beta_{n-2} \\
\vdots & \vdots & \ddots & & \vdots & \vdots \\
0 & 0 & 0 & \ddots & 0 & -\beta_{2} \\
0 & 0 & 0 & \ldots & 1 & -\beta_{1}
\end{array}\right]
$$

that is, a $n \times n$ matrix $C$ with $n>1$ is called a doubly companion matrix if its entries $c_{i j}$ satisfy $c_{i j}=1$ for all entries in the sub-maindiagonal of $C$ and else $c_{i j}=0$ for $i \neq 1$ and $j \neq n$.

We define a doubly Leslie matrix analogous as the doubly companion matrix by replacing the subdiagonal of the doubly companion matrix by $s_{1}, s_{2}, \ldots, s_{n-1}$ where $s_{j}, j=1,2, \ldots, n-1$, respectively, and denoted by $L$, that is, a doubly 
Leslie matrix is defined to be a matrix as follows:

$$
L=\left[\begin{array}{cccccc}
-a_{1} & -a_{2} & -a_{3} & \ldots & -a_{n-1} & -a_{n}-b_{n} \\
s_{1} & 0 & 0 & \ldots & 0 & -b_{n-1} \\
0 & s_{2} & 0 & \ldots & 0 & -b_{n-2} \\
0 & 0 & \ddots & \ddots & \vdots & \vdots \\
\vdots & \vdots & \ddots & \ddots & 0 & -b_{2} \\
0 & 0 & \cdots & 0 & s_{n-1} & -b_{1}
\end{array}\right],
$$

where $a_{j}, b_{j} \in \mathbb{R}$, the real numbers, $j=1,2, \ldots, n$. As the Leslie matrix, we restriction only $s_{j}>0, j=1,2, \ldots, n-1$.

For convenience, we can be written the matrix $L$ in a partitioned form as

$$
L=\left[\begin{array}{cc}
-\mathbf{p}^{T} & -a_{n}-b_{n} \\
\Lambda & -\mathbf{q}
\end{array}\right]_{(n, n)} \text { where } \mathbf{p}=\left[\begin{array}{c}
a_{1} \\
a_{2} \\
\vdots \\
a_{n-1}
\end{array}\right], \mathbf{q}=\left[\begin{array}{c}
b_{n-1} \\
b_{n-2} \\
\vdots \\
b_{1}
\end{array}\right]
$$

and $\Lambda=\operatorname{diag}\left(s_{1}, s_{2}, \ldots, s_{n-1}\right)$ is a diagonal matrix of order $n-1$.

We also define a rectangular doubly Leslie matrix of order $m \times n$, where $m=n-k$ and $1<k<n$ as follows:

$$
R=\left[\begin{array}{cccccc}
-a_{1} & -a_{2} & -a_{3} & \ldots & -a_{n-1} & -a_{n}-b_{n} \\
s_{1} & 0 & 0 & \cdots & 0 & -b_{n-1} \\
0 & s_{2} & 0 & \cdots & 0 & -b_{n-2} \\
\vdots & \ddots & \ddots & \ddots & \vdots & \vdots \\
0 & \cdots & 0 & s_{n-k} & 0 & -b_{k}
\end{array}\right]_{(m, n)}
$$

For convenience, we can be written the matrix $L$ in a partitioned form as

$$
R=\left[\begin{array}{cc}
-\mathbf{p}^{T} & -a_{n}-b_{n} \\
\Lambda_{k} & -\mathbf{q}_{k}
\end{array}\right]_{(m, n)} \quad \text { where } \mathbf{p}=\left[\begin{array}{c}
a_{1} \\
a_{2} \\
\vdots \\
a_{n-1}
\end{array}\right], \mathbf{q}_{k}=\left[\begin{array}{c}
b_{n-1} \\
b_{n-2} \\
\vdots \\
b_{k}
\end{array}\right]
$$

and $\Lambda_{k}=\left[\operatorname{diag}\left(s_{1}, s_{2}, \ldots, s_{n-k}\right) \mid 0\right]$ is a $(n-k) \times(n-1)$ block matrix which the first block is a diagonal matrix $\operatorname{diag}\left(s_{1}, s_{2}, \ldots, s_{n-k}\right)$ and the remainder block is a zero matrix of appropriated size.

We abbreviate doubly Leslie matrix to DLM and rectangular doubly Leslie matrix by RDLM. 
Let $M$ be a matrix partitioned into four blocks

$$
M=\left[\begin{array}{ll}
A & B \\
C & D
\end{array}\right]
$$

where the submatrix $C$ is assumed to be square and nonsingular. Brezinski in $[3$, p.232] asserted that, the Schur complement of $C$ in $M$, denoted by $(M / C)$, is defined by

$$
(M / C)=B-A C^{-1} D .
$$

As in (6), the Schur complement of $\Lambda$ in $L$, denoted by $(L / \Lambda)$, is a $1 \times 1$ matrix or a scalar

$$
\begin{aligned}
(L / \Lambda) & =\left(-a_{n}-b_{n}\right)-\left(-\mathbf{p}^{T}\right) \Lambda^{-1}(-\mathbf{q}) \\
& =-\left(\left(a_{n}+b_{n}\right)+\sum_{i=1}^{n-1} \frac{a_{i} b_{n-i}}{s_{i}}\right) .
\end{aligned}
$$

The author [7] asserted some basic properties of doubly Leslie matrix as in the following lemma.

Lemma 1. Let $L$ be a doubly Leslie matrix as in (3) with partitioned as

$$
L=\left[\begin{array}{cc}
-\mathbf{p}^{T} & -a_{n}-b_{n} \\
\Lambda & -\mathbf{q}
\end{array}\right]_{(n, n)}
$$

where $\mathbf{p}=\left[\begin{array}{llll}a_{1} & a_{2} & \ldots & a_{n-1}\end{array}\right]^{T}, \mathbf{q}=\left[\begin{array}{llll}b_{n-1} & b_{n-2} & \ldots & b_{1}\end{array}\right]^{T}$, and $\Lambda=$ $\operatorname{diag}\left(s_{1}, s_{2}, \ldots, s_{n-1}\right), s_{j}>0, j=1,2, \ldots, n-1$ is a diagonal matrix of order $n-1$, then

$$
\operatorname{det} L=(-1)^{n}\left(\left(a_{n}+b_{n}\right)+\sum_{i=1}^{n-1} \frac{a_{i} b_{n-i}}{s_{i}}\right) \prod_{i=1}^{n-1} s_{i}
$$

and, if $\operatorname{det} L \neq 0$ then

$$
L^{-1}=(L / \Lambda)^{-1}\left[\begin{array}{cc}
\Lambda^{-1} \mathbf{q} & (L / \Lambda) \Lambda^{-1}+\left(\Lambda^{-1} \mathbf{q p}^{T} \Lambda^{-1}\right) \\
1 & \mathbf{p}^{T} \Lambda^{-1}
\end{array}\right]_{(n, n)}
$$

where $(L / \Lambda)=-\left(\left(a_{n}+b_{n}\right)+\sum_{i=1}^{n-1} \frac{a_{i} b_{n-i}}{s_{i}}\right)$, as in $(7)$, and $\Lambda^{-1}=$ $\operatorname{diag}\left(\frac{1}{s_{1}}, \frac{1}{s_{2}}, \ldots, \frac{1}{s_{n-1}}\right)$.

In the present paper we give explicit Moore-Penrose inverse and group inverse formulae for the doubly Leslie matrix and give some related topics. 


\section{Preliminaries}

Let $\mathbb{R}^{m \times n}$ denote the set of all $m \times n$ matrices over the field of real numbers $\mathbb{R}$. The Moore-Penrose inverse of a matrix $A \in \mathbb{R}^{m \times n}$ is the unique matrix $X \in \mathbb{R}^{n \times m}$ satisfying the four Penrose conditions

$$
A=A X A, \quad X=X A X, \quad(A X)^{T}=A X \quad \text { and } \quad(X A)^{T}=X A
$$

and is denoted by $A^{\dagger}$. The group inverse of a matrix $A \in \mathbb{R}^{n \times n}$ is the unique matrix $X \in \mathbb{R}^{n \times n}$ satisfying

$$
A=A X A, \quad X=X A X \quad \text { and } \quad A X=X A
$$

and is denoted by $A^{\sharp}$. A well known characterization for the existence of $A^{\sharp}$ is that $\operatorname{rank}(A)=\operatorname{rank}\left(A^{2}\right)$, [1]. If $A$ is nonsingular, then $A^{-1}=A^{\dagger}=A^{\sharp}$. Recall that $A \in \mathbb{R}^{n \times n}$ is called range-symmetric if $\operatorname{range}(A)=\operatorname{range}\left(A^{T}\right)$. If $A$ is range-symmetric, then $A^{\dagger}=A^{\sharp}$.

A system of linear equation $A \mathrm{x}=\mathrm{b}$ need not possess a solution when $\operatorname{rank}(A) \neq \operatorname{rank}[A: \mathbf{b}]$. That is $\mathbf{b}$ is not in the range of $A$. The Moore-Penrose inverse is most often used to solve least squares systems. It is still desirable to to find a $\mathbf{x}_{0}$ that is closest to a solution. The residual vector is a key component to solve these systems.

Theorem 2 ([1]). Let $A \in \mathbb{R}^{m \times n}$ with $\operatorname{rank}(A)=r \neq 0$, and suppose $A=F G$ is a full rank factorization of $A$. Then

1. $F^{\dagger}=\left(F^{T} F\right)^{-1} F^{T}$,

2. $F^{\dagger} F=I_{r}$, the $r \times r$ identity matrix,

3. $G^{\dagger}=G^{T}\left(G G^{T}\right)^{-1}$,

4. $G G^{\dagger}=I_{r}$,

5. $A^{\dagger}=G^{\dagger} F^{\dagger}$.

More generally, for any $m \times n$ matrix $A$ of full row $\operatorname{rank} m, A=I_{m} A$ is a full rank factorization of $A$. Then

$$
A^{\dagger}=A^{T}\left(A A^{T}\right)^{-1} .
$$

The group inverse is very useful and has applications in many fields such as singular differential and difference equations, Markov chains, and iterative methods, see for instance [1]. 
Theorem 3 ([1]). Let a square matrix $A$ have the full rank factorization $A=F G$. Then $A$ has a group inverse if and only if $G F$ is nonsingular. In which case,

$$
A^{\sharp}=F(G F)^{-2} G \text {. }
$$

\section{Moore-Penrose Inverse of RDLM}

Penrose [5, p.18]. It is possible to calculate $A^{\dagger}$ even when $A^{*} A$ and $A A^{*}$ are both singular by the following methods, where $A^{*}$ is the conjugate transpose of the matrix $A$.

Any matrix $M$ can be partitioned in the form

$$
M=\left[\begin{array}{ll}
A & B \\
C & D
\end{array}\right]
$$

where $D=C A^{-1} B$, (using a suitable arrangement of rows and columns). $A$ being any non-singular submatrix whose rank is equal to that of the whole matrix. It is then easily verified that

$$
M^{\dagger}=\left[\begin{array}{cc}
A & B \\
C & D
\end{array}\right]^{\dagger}=\left[\begin{array}{cc}
A^{*} K A^{*} & A^{*} K C^{*} \\
B^{*} K A^{*} & B^{*} K C^{*}
\end{array}\right],
$$

where $K=\left(A A^{*}+B B^{*}\right)^{-1} A\left(A^{*} A+C^{*} C\right)^{-1}$. The matrices $A A^{*}+B B^{*}$ and $A^{*} A+C^{*} C$ are positive definite, since $A$ is non-singular. Thus the generalized inverse of any matrix can be expressed in terms of ordinary reciprocals of matrices.

We have the following main results.

Lemma 4. If $A=P B$, where $P$ is a permutation matrix, then

$$
A^{\dagger}=B^{\dagger} P^{T}
$$

Proof. It is straightforward to verify that $B^{\dagger} P^{T}$ satisfies the four Penrose conditions. Clearly:

1. $P B\left(B^{\dagger} P^{T}\right) P B=P B B^{\dagger} B=P B$,

2. $\left(B^{\dagger} P^{T}\right) P B\left(B^{\dagger} P^{T}\right)=B^{\dagger} B B^{\dagger} P^{T}=B^{\dagger} P^{T}$,

3. $\left[P B\left(B^{\dagger} P^{T}\right)\right]^{T}=\left[P B B^{\dagger} P^{T}\right]^{T}=P\left(B B^{\dagger}\right)^{T} P^{T}=P B B^{\dagger} P^{T}=P B\left(B^{\dagger} P^{T}\right)$, 
4. $\left[\left(B^{\dagger} P^{T}\right) P B\right]^{T}=\left[B^{\dagger} P^{T} P B\right]^{T}=\left[B^{\dagger} B\right]^{T}=B^{\dagger} B=B^{\dagger}\left(P^{T} P\right) B$

$$
=\left(B^{\dagger} P^{T}\right) P B \text {. }
$$

Lemma 5. For an $m \times n \mathbb{R}$-matrix $N$ of $\operatorname{rank} r<\min (m, n)$, and $N$ partitioned in the form

$$
N=\left[\begin{array}{ll}
A & B \\
C & D
\end{array}\right]
$$

where $C$ is $r \times r$ nonsingular. Then

$$
N^{\dagger}=\left[\begin{array}{ll}
C^{T} K D^{T} & C^{T} K C^{T} \\
D^{T} K A^{T} & D^{T} K C^{T}
\end{array}\right]
$$

where $K=\left(C C^{T}+D D^{T}\right)^{-1} C\left(C^{T} C+A^{T} A\right)^{-1}$. The matrices $C C^{T}+D D^{T}$ and $C^{T} C+A^{T} A$ are positive definite, since $C$ is non-singular.

Proof. Let $P=\left[\begin{array}{cc}0 & I_{r} \\ I_{m-r} & 0\end{array}\right]_{(m \times m)}$ be a permutation matrix. Premultiplying the matrix $N$ by $P$.

$$
P N=\left[\begin{array}{ll}
C & D \\
A & B
\end{array}\right]
$$

Since $P$ is a unitary matrix and by (12). We have

$$
(P N)^{\dagger}=N^{\dagger} P^{T} \text {. }
$$

Therefore

$$
(P N)^{\dagger}=\left[\begin{array}{cc}
C & D \\
A & B
\end{array}\right]^{\dagger}=N^{\dagger} P^{T}
$$

and

$$
N^{\dagger}=\left[\begin{array}{ll}
C & D \\
A & B
\end{array}\right]^{\dagger} P
$$

As in (11), we have

$$
\left[\begin{array}{ll}
C & D \\
A & B
\end{array}\right]^{\dagger}=\left[\begin{array}{cc}
C^{T} K C^{T} & C^{T} K A^{T} \\
D^{T} K C^{T} & D^{T} K A^{T}
\end{array}\right]
$$

where $K=\left(C C^{T}+D D^{T}\right)^{-1} C\left(C^{T} C+A^{T} A\right)^{-1}$. The matrices $C C^{T}+D D^{T}$ and $C^{T} C+A^{T} A$ are positive definite, since $C$ is non-singular. Therefore $C C^{T}+D D^{T}$ and $C^{T} C+A^{T} A$ are also non-singular matrices. We have

$$
N^{\dagger}=\left[\begin{array}{ll}
C^{T} K C^{T} & C^{T} K A^{T} \\
D^{T} K C^{T} & D^{T} K A^{T}
\end{array}\right] P=\left[\begin{array}{cc}
C^{T} K A^{T} & C^{T} K C^{T} \\
D^{T} K A^{T} & D^{T} K C^{T}
\end{array}\right] .
$$

The proof is complete. 
Theorem 6. Let $L$ be a doubly Leslie matrix as in (3) with partitioned as

$$
L=\left[\begin{array}{cc}
-\mathbf{p}^{T} & -a_{n}-b_{n} \\
\Lambda & -\mathbf{q}
\end{array}\right]_{(n, n)}
$$

where $\mathbf{p}=\left[\begin{array}{llll}a_{1} & a_{2} & \ldots & a_{n-1}\end{array}\right]^{T}, \mathbf{q}=\left[\begin{array}{llll}b_{n-1} & b_{n-2} & \ldots & b_{1}\end{array}\right]^{T}$, and $\Lambda=$ $\operatorname{diag}\left(s_{1}, s_{2}, \ldots, s_{n-1}\right), s_{j}>0, j=1,2, \ldots, n-1$ is a diagonal matrix of order $n-1$, then

$$
L^{\dagger}=\left[\begin{array}{ll}
-\Lambda K \mathbf{p} & \Lambda K \Lambda \\
\mathbf{q}^{T} K \mathbf{p} & -\mathbf{q}^{T} K \Lambda
\end{array}\right],
$$

where $K=\left(\Lambda^{2}+\mathbf{q q}^{T}\right)^{-1} \Lambda\left(\Lambda^{2}+\mathbf{p p}^{T}\right)^{-1}$.

Proof. If $\operatorname{det} L \neq 0$ then $L^{\dagger}=L^{-1}$ which appeared in (9).

In general

$$
\begin{aligned}
L^{\dagger} & =\left[\begin{array}{cc}
A & B \\
C & D
\end{array}\right]^{\dagger}=\left[\begin{array}{cc}
C^{T} K A^{T} & C^{T} K C^{T} \\
D^{T} K A^{T} & D^{T} K C^{T}
\end{array}\right] \\
& =\left[\begin{array}{ll}
\Lambda^{T} K\left(-\mathbf{p}^{T}\right)^{T} & \Lambda^{T} K \Lambda^{T} \\
(-\mathbf{q})^{T} K\left(-\mathbf{p}^{T}\right)^{T} & (-\mathbf{q})^{T} K \Lambda^{T}
\end{array}\right] \\
& =\left[\begin{array}{ll}
-\Lambda K \mathbf{p} & \Lambda K \Lambda \\
\mathbf{q}^{T} K \mathbf{p} & -\mathbf{q}^{T} K \Lambda
\end{array}\right]
\end{aligned}
$$

where

$$
\begin{aligned}
K & =\left(\Lambda \Lambda^{T}+(-\mathbf{q})(-\mathbf{q})^{T}\right)^{-1} \Lambda\left(\Lambda^{T} \Lambda+\left(-\mathbf{p}^{T}\right)^{T}\left(-\mathbf{p}^{T}\right)\right)^{-1} \\
& =\left(\Lambda^{2}+\mathbf{q} \mathbf{q}^{T}\right)^{-1} \Lambda\left(\Lambda^{2}+\mathbf{p p}^{T}\right)^{-1}
\end{aligned}
$$

Corollary 7. Let $R$ be a rectangle doubly Leslie matrix as in (5) with partitioned as

$$
R=\left[\begin{array}{cc}
-\mathbf{p}^{T} & -a_{n}-b_{n} \\
\Lambda_{k} & -\mathbf{q}_{k}
\end{array}\right]_{(m, n)}
$$

where $m=n-k$,

$$
\mathbf{p}=\left[\begin{array}{llll}
a_{1} & a_{2} & \ldots & a_{n-1}
\end{array}\right]^{T}, \quad \mathbf{q}=\left[\begin{array}{llll}
b_{n-1} & b_{n-2} & \ldots & b_{k}
\end{array}\right]^{T},
$$

and $\Lambda_{k}=\left[\operatorname{diag}\left(s_{1}, s_{2}, \ldots, s_{n-k}\right) \mid 0\right]$ is a $(n-k) \times(n-1)$ block matrix, then

$$
L^{\dagger}=\left[\begin{array}{ll}
-\Lambda_{k} K \mathbf{p} & \Lambda_{k} K \Lambda_{k} \\
\mathbf{q}_{k}^{T} K \mathbf{p} & -\mathbf{q}_{k}^{T} K \Lambda_{k}
\end{array}\right]
$$

where $K=\left(\Lambda_{k} \Lambda_{k}^{T}+\mathbf{q}_{k} \mathbf{q}_{k}^{T}\right)^{-1} \Lambda_{k}\left(\Lambda_{k}^{T} \Lambda_{k}+\mathbf{p p}^{T}\right)^{-1}$. 
Proof. The proof is an analogous as in Theorem 6 .

Let's consider some examples.

EXAMPLE

$$
L=\left[\begin{array}{rrrr}
1 & 2 & -1 & -3 \\
1 & 0 & 0 & -1 \\
0 & 2 & 0 & 0 \\
0 & 0 & 1 & 2
\end{array}\right]=:\left[\begin{array}{cc}
-\mathbf{p}^{T} & -a_{n}-b_{n} \\
\Lambda & -\mathbf{q}
\end{array}\right]
$$

where $\mathbf{p}=\left[\begin{array}{lll}-1 & -2 & 1\end{array}\right]^{T}, \mathbf{q}=\left[\begin{array}{lll}1 & 0 & -2\end{array}\right]^{T}$, and $\Lambda=\operatorname{diag}(1,2,1)$, is a diagonal matrix of order 3 , then

$$
L^{\dagger}=\left[\begin{array}{ll}
-\Lambda K \mathbf{p} & \Lambda K \Lambda \\
\mathbf{q}^{T} K \mathbf{p} & -\mathbf{q}^{T} K \Lambda
\end{array}\right]
$$

where $K=\left(\Lambda^{2}+\mathbf{q q}^{T}\right)^{-1} \Lambda\left(\Lambda^{2}+\mathbf{p p}^{T}\right)^{-1}$.

First we calculate $\mathbf{q q}^{T}$ and $\mathbf{p} \mathbf{p}^{T}$.

$$
\begin{aligned}
\mathbf{q q}^{T} & =\left[\begin{array}{r}
1 \\
0 \\
-2
\end{array}\right]\left[\begin{array}{lll}
1 & 0 & -2
\end{array}\right]=\left[\begin{array}{rrr}
1 & 0 & -2 \\
0 & 0 & 0 \\
-2 & 0 & 4
\end{array}\right], \\
\mathbf{p p}^{T} & =\left[\begin{array}{r}
-1 \\
-2 \\
1
\end{array}\right]\left[\begin{array}{lll}
-1 & -2 & 1
\end{array}\right]=\left[\begin{array}{rrr}
1 & 2 & -1 \\
2 & 4 & -2 \\
-1 & -2 & 1
\end{array}\right],
\end{aligned}
$$

and

$$
\begin{aligned}
& \left(\Lambda^{2}+\mathbf{q q}^{T}\right)^{-1}=\left[\begin{array}{rrr}
2 & 0 & -2 \\
0 & 4 & 0 \\
-2 & 0 & 5
\end{array}\right]=\left[\begin{array}{rrr}
\frac{5}{6} & 0 & \frac{1}{3} \\
0 & \frac{1}{4} & 0 \\
\frac{1}{3} & 0 & \frac{1}{3}
\end{array}\right], \\
& \left(\Lambda^{2}+\mathbf{p p}^{T}\right)^{-1}=\left[\begin{array}{rrrr}
2 & 2 & -1 \\
2 & 8 & -2 \\
-1 & -2 & 2
\end{array}\right]=\left[\begin{array}{rrr}
\frac{3}{4} & -\frac{1}{8} & \frac{1}{4} \\
-\frac{1}{8} & \frac{3}{16} & \frac{1}{8} \\
\frac{1}{4} & \frac{1}{8} & \frac{3}{4}
\end{array}\right],
\end{aligned}
$$

we have

$$
K=\left(\Lambda^{2}+\mathbf{q q}^{T}\right)^{-1} \Lambda\left(\Lambda^{2}+\mathbf{p} \mathbf{p}^{T}\right)^{-1}=\left[\begin{array}{ccc}
\frac{17}{24} & -\frac{1}{16} & \frac{11}{24} \\
-\frac{1}{16} & \frac{3}{32} & \frac{1}{16} \\
\frac{1}{3} & 0 & \frac{1}{3}
\end{array}\right]
$$


Finally,

$$
\begin{aligned}
& -\Lambda K \mathbf{p}=-\left[\begin{array}{lll}
1 & 0 & 0 \\
0 & 2 & 0 \\
0 & 0 & 1
\end{array}\right]\left[\begin{array}{ccc}
\frac{17}{24} & -\frac{1}{16} & \frac{11}{24} \\
-\frac{1}{16} & \frac{3}{32} & \frac{1}{16} \\
\frac{1}{3} & 0 & \frac{1}{3}
\end{array}\right]\left[\begin{array}{r}
-1 \\
-2 \\
1
\end{array}\right] \\
& =\left[\begin{array}{l}
\frac{1}{8} \\
\frac{1}{8} \\
0
\end{array}\right] \\
& \Lambda K \Lambda=\left[\begin{array}{lll}
1 & 0 & 0 \\
0 & 2 & 0 \\
0 & 0 & 1
\end{array}\right]\left[\begin{array}{ccc}
\frac{17}{24} & -\frac{1}{16} & \frac{11}{24} \\
-\frac{1}{16} & \frac{3}{32} & \frac{1}{16} \\
\frac{1}{3} & 0 & \frac{1}{3}
\end{array}\right]\left[\begin{array}{lll}
1 & 0 & 0 \\
0 & 2 & 0 \\
0 & 0 & 1
\end{array}\right] \\
& =\left[\begin{array}{ccc}
\frac{17}{24} & -\frac{1}{8} & \frac{11}{24} \\
-\frac{1}{8} & \frac{3}{8} & \frac{1}{8} \\
\frac{1}{3} & 0 & \frac{1}{3}
\end{array}\right] \\
& \mathbf{q}^{T} K \mathbf{p}=\left[\begin{array}{lll}
1 & 0 & -2
\end{array}\right]\left[\begin{array}{ccc}
\frac{17}{24} & -\frac{1}{16} & \frac{11}{24} \\
-\frac{1}{16} & \frac{3}{32} & \frac{1}{16} \\
\frac{1}{3} & 0 & \frac{1}{3}
\end{array}\right]\left[\begin{array}{r}
-1 \\
-2 \\
1
\end{array}\right]=-\frac{1}{8} \\
& -\mathbf{q}^{T} K \Lambda=-\left[\begin{array}{lll}
1 & 0 & -2
\end{array}\right]\left[\begin{array}{ccc}
\frac{17}{24} & -\frac{1}{16} & \frac{11}{24} \\
-\frac{1}{16} & \frac{3}{32} & \frac{1}{16} \\
\frac{1}{3} & 0 & \frac{1}{3}
\end{array}\right]\left[\begin{array}{lll}
1 & 0 & 0 \\
0 & 2 & 0 \\
0 & 0 & 1
\end{array}\right] \\
& =\left[\begin{array}{lll}
-\frac{1}{24} & \frac{1}{8} & \frac{5}{24}
\end{array}\right] \text {. }
\end{aligned}
$$

Therefore

$$
L^{\dagger}=\left[\begin{array}{ll}
-\Lambda K \mathbf{p} & \Lambda K \Lambda \\
\mathbf{q}^{T} K \mathbf{p} & -\mathbf{q}^{T} K \Lambda
\end{array}\right]=\left[\begin{array}{cccc}
\frac{1}{8} & \frac{17}{24} & -\frac{1}{8} & \frac{11}{24} \\
\frac{1}{8} & -\frac{1}{8} & \frac{3}{8} & \frac{1}{8} \\
0 & \frac{1}{3} & 0 & \frac{1}{3} \\
-\frac{1}{8} & -\frac{1}{24} & \frac{1}{8} & \frac{5}{24}
\end{array}\right]
$$

This matrix is satisfies the four Penrose conditions.

ExAMPLE. For a full row rank rectangle doubly Leslie matrix of order $3 \times 4$

$$
R=\left[\begin{array}{rrrr}
1 & 2 & -1 & -3 \\
1 & 0 & 0 & -1 \\
0 & 2 & 0 & 0
\end{array}\right]
$$


From (10),

$$
\begin{aligned}
& R^{\dagger}=R^{T}\left(R R^{T}\right)^{-1} \\
& =\left[\begin{array}{ccc}
1 & 1 & 0 \\
2 & 0 & 2 \\
-1 & 0 & 0 \\
-3 & -1 & 0
\end{array}\right]\left(\left[\begin{array}{rrrr}
1 & 2 & -1 & -3 \\
1 & 0 & 0 & -1 \\
0 & 2 & 0 & 0
\end{array}\right]\left[\begin{array}{ccc}
1 & 1 & 0 \\
2 & 0 & 2 \\
-1 & 0 & 0 \\
-3 & -1 & 0
\end{array}\right]\right) \\
& =\left[\begin{array}{ccc}
1 & 1 & 0 \\
2 & 0 & 2 \\
-1 & 0 & 0 \\
-3 & -1 & 0
\end{array}\right]\left[\begin{array}{ccc}
\frac{1}{3} & -\frac{2}{3} & -\frac{1}{3} \\
-\frac{2}{3} & \frac{11}{6} & \frac{2}{3} \\
-\frac{1}{3} & \frac{2}{3} & \frac{7}{12}
\end{array}\right] \\
& =\left[\begin{array}{ccc}
-\frac{1}{3} & \frac{7}{6} & \frac{1}{3} \\
0 & 0 & \frac{1}{2} \\
-\frac{1}{3} & \frac{2}{3} & \frac{1}{3} \\
-\frac{1}{3} & \frac{1}{6} & \frac{1}{3}
\end{array}\right]
\end{aligned}
$$

This matrix is also satisfies the four Penrose conditions.

\section{Group Inverse of DLM}

As in [1, p.167] we have the following useful result.

Theorem 8. Let $A$ be a square singular matrix, $\operatorname{rank} A=\operatorname{rank} A^{2}$, and $R(A)$ be the range of $A$. If the system

$$
A \mathbf{x}=\mathbf{b}, \quad \mathbf{x} \in R(A)
$$

has a solution, it is uniquely given by

$$
\mathbf{x}=A^{\sharp} \mathbf{b} .
$$

Proof. Suppose that $\mathbf{x} \in R(A)$ where $R(A)$ is the range of $A$. There is a vector $\mathbf{y}$ such that $A \mathbf{y}=\mathbf{x}$. Let a solution $\mathbf{x}$ be written as $\mathbf{x}=A \mathbf{y}_{1}$ for some $\mathbf{y}_{1}$. We have

$$
A \mathbf{x}=A A \mathbf{y}_{1}=A^{2} \mathbf{y}_{1}
$$

then $A^{2} \mathbf{y}_{1}=\mathbf{b}$. Since $\operatorname{rank} A=\operatorname{rank} A^{2}$, there is a unique $A^{\sharp}$ such that

$$
A A^{\sharp} A=A, \quad A^{\sharp} A A^{\sharp}=A^{\sharp}, \quad \text { and } \quad A A^{\sharp}=A^{\sharp} A \text {. }
$$


Therefore

$$
\begin{aligned}
\mathbf{x} & =A \mathbf{y}_{1} \\
& =A A^{\sharp} A \mathbf{y}_{1} \\
& =A^{2} A^{\sharp} \mathbf{y}_{1} \\
& =A^{\sharp} A^{2} \mathbf{y}_{1} \\
& =A^{\sharp} A \mathbf{x} \\
& =A^{\sharp} \mathbf{b} .
\end{aligned}
$$

Let $L$ be a doubly Leslie matrix as in (3) with partitioned as

$$
L=\left[\begin{array}{cc}
-\mathbf{p}^{T} & -a_{n}-b_{n} \\
\Lambda & -\mathbf{q}
\end{array}\right]_{(n, n)}
$$

If $\operatorname{det} L \neq 0$ then $L^{\sharp}=L^{-1}$ which was shown in (9). We interested in study the only case $\operatorname{rank}(L) \neq n$. By the definition of DLM the rank of $L$ is at least $n-1$. Since equivalence matrix has the same rank, we reduce the matrix $L$ to a reduced echelon form as follows:

$$
\begin{aligned}
& {\left[\begin{array}{ccccc}
0 & \frac{1}{s_{1}} & 0 & \cdots & 0 \\
0 & 0 & \frac{1}{s_{2}} & \ddots & \vdots \\
\vdots & \vdots & \ddots & \ddots & 0 \\
0 & 0 & \cdots & 0 & \frac{1}{s_{n-1}} \\
1 & \frac{a_{1}}{s_{1}} & \frac{a_{2}}{s_{2}} & \cdots & \frac{a_{n-1}}{s_{n-1}}
\end{array}\right]\left[\begin{array}{ccccc}
-a_{1} & -a_{2} & \cdots & -a_{n-1} & -a_{n}-b_{n} \\
s_{1} & 0 & \cdots & 0 & -b_{n-1} \\
0 & s_{2} & \ddots & \vdots & \vdots \\
\vdots & \ddots & \ddots & 0 & -b_{2} \\
0 & \cdots & 0 & s_{n-1} & -b_{1}
\end{array}\right]} \\
& =\left[\begin{array}{ccccc}
1 & 0 & \cdots & 0 & -\frac{b_{n-1}}{s_{1}} \\
0 & 1 & 0 & \vdots & -\frac{b_{n-2}}{s_{2}} \\
0 & 0 & \ddots & 0 & \vdots \\
\vdots & \vdots & \ddots & 1 & -\frac{b_{1}}{s_{n-1}} \\
0 & 0 & \cdots & 0 & -a_{n}-b_{n}-\frac{a_{1}}{s_{1}} b_{n-1}-\frac{a_{2}}{s_{2}} b_{n-2}-\cdots-\frac{a_{n-2}}{s_{n-2}} b_{2}-\frac{a_{n-1}}{s_{n-1}} b_{1}
\end{array}\right]
\end{aligned}
$$

We see that $\operatorname{rank}(L)=n-1$ if and only if

$$
-a_{n}-b_{n}-\frac{a_{1}}{s_{1}} b_{n-1}-\frac{a_{2}}{s_{2}} b_{n-2}-\cdots-\frac{a_{n-2}}{s_{n-2}} b_{2}-\frac{a_{n-1}}{s_{n-1}} b_{1}=0
$$

if and only if

$$
-a_{n}-b_{n}=\frac{a_{1}}{s_{1}} b_{n-1}+\frac{a_{2}}{s_{2}} b_{n-2}+\cdots+\frac{a_{n-2}}{s_{n-2}} b_{2}+\frac{a_{n-1}}{s_{n-1}} b_{1} .
$$


We factor $L$ to full rank factorization as follows:

$$
\begin{aligned}
L=F G & =\left[\begin{array}{cccc}
-a_{1} & -a_{2} & \cdots & -a_{n-1} \\
s_{1} & 0 & \cdots & 0 \\
0 & s_{2} & \ddots & \vdots \\
\vdots & \ddots & \ddots & 0 \\
0 & \cdots & 0 & s_{n-1}
\end{array}\right]\left[\begin{array}{ccccc}
1 & 0 & \cdots & 0 & -\frac{b_{n-1}}{s_{1}} \\
0 & 1 & \ddots & \vdots & -\frac{b_{n-2}}{s_{2}} \\
\vdots & \ddots & \ddots & 0 & \vdots \\
0 & \cdots & 0 & 1 & -\frac{b_{1}}{s_{n-1}}
\end{array}\right] \\
& =\left[\begin{array}{c}
-\mathbf{p}^{T} \\
\Lambda
\end{array}\right]\left[\begin{array}{ll}
I_{n-1} & -\mathbf{q}_{1}
\end{array}\right],
\end{aligned}
$$

where $\mathbf{p}=\left[\begin{array}{c}a_{1} \\ a_{2} \\ \vdots \\ a_{n-1}\end{array}\right], \mathbf{q}_{1}=\left[\begin{array}{c}\frac{b_{n-1}}{s_{1}} \\ \frac{b_{n-2}}{s_{2}} \\ \vdots \\ \frac{b_{1}}{s_{n-1}}\end{array}\right]$, and $\Lambda=\operatorname{diag}\left(s_{1}, s_{2}, \ldots, s_{n-1}\right)$.

Also, by direct computation, we have

$$
G F=\left[\begin{array}{cccccc}
-a_{1} & -a_{2} & -a_{3} & \ldots & -a_{n-2} & -a_{n-1}-b_{n-1} \frac{s_{n-1}}{s_{1}} \\
s_{1} & 0 & 0 & \ldots & 0 & -b_{n-2} \frac{s_{n-1}}{s_{2}} \\
0 & s_{2} & 0 & \ldots & 0 & -b_{n-3} \frac{s_{n-1}}{s_{3}} \\
0 & 0 & \ddots & \ddots & \vdots & \vdots \\
\vdots & \vdots & \ddots & \ddots & 0 & -b_{2} \frac{s_{n-1}}{s_{n-2}} \\
0 & 0 & \ldots & 0 & s_{n-2} & -b_{1} \frac{s_{n-1}}{s_{n-1}}
\end{array}\right]=: M
$$

The matrix $G F=: M$ is a doubly Leslie matrix of order $(n-1) \times(n-1)$.

$$
M=\left[\begin{array}{cc}
-\mathbf{p}_{1}^{T} & -a_{n-1}-b_{n-1} \frac{s_{n-1}}{s_{1}} \\
\Lambda_{1} & -\mathbf{q}_{2}
\end{array}\right]_{(n-1, n-1)},
$$

where $\mathbf{p}_{1}=\left[\begin{array}{c}a_{1} \\ a_{2} \\ \vdots \\ a_{n-2}\end{array}\right], \mathbf{q}_{2}=\left[\begin{array}{c}b_{n-2} \frac{s_{n-1}}{s_{2}} \\ b_{n-3} \frac{s_{n-1}}{s_{3}} \\ \vdots \\ b_{1} \frac{s_{n-1}}{s_{n-1}}\end{array}\right]$, and

$$
\Lambda_{1}=\operatorname{diag}\left(s_{1}, s_{2}, \ldots, s_{n-2}\right)
$$

is a diagonal matrix of order $n-1$.

By (9), we have

$$
M^{-1}=\left(M / \Lambda_{1}\right)^{-1}\left[\begin{array}{cc}
\Lambda_{1}^{-1} \mathbf{q}_{2} & \left(M / \Lambda_{1}\right) \Lambda_{1}^{-1}+\left(\Lambda_{1}^{-1} \mathbf{q}_{2} \mathbf{p}_{1}^{T} \Lambda_{1}^{-1}\right) \\
1 & \mathbf{p}_{1}^{T} \Lambda_{1}^{-1}
\end{array}\right]
$$


where $\left(M / \Lambda_{1}\right)=-\left(\left(a_{n-1}+b_{n-1} \frac{s_{n-1}}{s_{1}}\right)+s_{n-1} \sum_{i=1}^{n-2} \frac{a_{i} b_{n-i-1}}{s_{i} s_{i+1}}\right)$, as in $(7)$, and $\Lambda_{1}^{-1}=\operatorname{diag}\left(\frac{1}{s_{1}}, \frac{1}{s_{2}}, \ldots, \frac{1}{s_{n-2}}\right)$.

From Theorem 3, we have

$$
\begin{aligned}
& L^{\sharp}=F(G F)^{-2} G \\
& =\left[\begin{array}{c}
-\mathbf{p}^{T} \\
\Lambda
\end{array}\right] \times \\
& \left(\left(M / \Lambda_{1}\right)^{-1}\left[\begin{array}{cc}
\Lambda_{1}^{-1} \mathbf{q}_{2} & \left(M / \Lambda_{1}\right) \Lambda_{1}^{-1}+\left(\Lambda_{1}^{-1} \mathbf{q}_{2} \mathbf{p}_{1}^{T} \Lambda_{1}^{-1}\right) \\
1 & \mathbf{p}_{1}^{T} \Lambda_{1}^{-1}
\end{array}\right]\right)^{2} \times \\
& {\left[\begin{array}{ll}
I_{n-1} & -\mathbf{q}_{1}
\end{array}\right]} \\
& =\left(M / \Lambda_{1}\right)^{-2}\left[\begin{array}{c}
-\mathbf{p}^{T} \\
\Lambda
\end{array}\right] \times \\
& {\left[\begin{array}{cc}
\Lambda_{1}^{-1} \mathbf{q}_{2} & \left(M / \Lambda_{1}\right) \Lambda_{1}^{-1}+\left(\Lambda_{1}^{-1} \mathbf{q}_{2} \mathbf{p}_{1}^{T} \Lambda_{1}^{-1}\right) \\
1 & \mathbf{p}_{1}^{T} \Lambda_{1}^{-1}
\end{array}\right]^{2}\left[\begin{array}{cc}
I_{n-1} & -\mathbf{q}_{1}
\end{array}\right]}
\end{aligned}
$$

Let's consider the same example.

EXAMPLE

$$
L=\left[\begin{array}{rrrr}
1 & 2 & -1 & -3 \\
1 & 0 & 0 & -1 \\
0 & 2 & 0 & 0 \\
0 & 0 & 1 & 2
\end{array}\right]
$$

where $\mathbf{p}=\left[\begin{array}{lll}-1 & -2 & 1\end{array}\right]^{T}, \mathbf{q}=\left[\begin{array}{lll}1 & 0 & -2\end{array}\right]^{T}$, and $\Lambda=\operatorname{diag}(1,2,1)$, is a diagonal matrix of order 3 .

Since $\operatorname{det}(L)=0$, we have $\operatorname{rank}(L)=\operatorname{rank}\left(L^{2}\right)$, we know that the unique $L^{\sharp}$ exists. Now

$$
L=F G=\left[\begin{array}{rrr}
1 & 2 & -1 \\
1 & 0 & 0 \\
0 & 2 & 0 \\
0 & 0 & 1
\end{array}\right]\left[\begin{array}{rrrr}
1 & 0 & 0 & -1 \\
0 & 1 & 0 & 0 \\
0 & 0 & 1 & 2
\end{array}\right]
$$

and

$$
G F=\left[\begin{array}{cccc}
1 & 0 & 0 & -1 \\
0 & 1 & 0 & 0 \\
0 & 0 & 1 & 2
\end{array}\right]\left[\begin{array}{ccc}
1 & 2 & -1 \\
1 & 0 & 0 \\
0 & 2 & 0 \\
0 & 0 & 1
\end{array}\right]=\left[\begin{array}{ccc}
1 & 2 & -2 \\
1 & 0 & 0 \\
0 & 2 & 2
\end{array}\right]
$$




$$
\begin{aligned}
& (G F)^{-1}=\left[\begin{array}{cccc}
1 & 2 & -2 & -1 \\
1 & 0 & 0 \\
0 & 2 & 2
\end{array}\right]=\left[\begin{array}{ccc}
0 & 1 & 0 \\
\frac{1}{4} & -\frac{1}{4} & \frac{1}{4} \\
-\frac{1}{4} & \frac{1}{4} & \frac{1}{4}
\end{array}\right] \\
& (G F)^{-2}=\left[\begin{array}{ccc}
\frac{1}{4} & -\frac{1}{4} & \frac{1}{4} \\
-\frac{1}{8} & \frac{3}{8} & 0 \\
0 & -\frac{1}{4} & \frac{1}{8}
\end{array}\right] .
\end{aligned}
$$

Finally

$$
\begin{aligned}
L^{\sharp} & =F(G F)^{-2} G \\
= & {\left[\begin{array}{rrr}
1 & 2 & -1 \\
1 & 0 & 0 \\
0 & 2 & 0 \\
0 & 0 & 1
\end{array}\right]\left[\begin{array}{ccc}
\frac{1}{4} & -\frac{1}{4} & \frac{1}{4} \\
-\frac{1}{8} & \frac{3}{8} & 0 \\
0 & -\frac{1}{4} & \frac{1}{8}
\end{array}\right]\left[\begin{array}{cccc}
1 & 0 & 0 & -1 \\
0 & 1 & 0 & 0 \\
0 & 0 & 1 & 2
\end{array}\right] } \\
= & {\left[\begin{array}{ccccc}
0 & \frac{3}{4} & \frac{1}{8} & \frac{1}{4} \\
\frac{1}{4} & -\frac{1}{4} & \frac{1}{4} & \frac{1}{4} \\
-\frac{1}{4} & \frac{3}{4} & 0 & \frac{1}{4} \\
0 & -\frac{1}{4} & \frac{1}{8} & \frac{1}{4}
\end{array}\right] . }
\end{aligned}
$$

This matrix is satisfies the three conditions for group inverse.

\section{Conclusion}

In this paper, we mainly study about the explicit formula of Moore-Penrose inverse and group inverse of doubly Leslie matrix.

\section{Acknowledgments}

The author is very grateful to the anonymous referees for their comments and suggestions, which inspired the improvement of the manuscript. This work was supported by Naresuan University.

\section{References}

[1] A. Ben-Israel, T.N.E. Greville, Generalized Inverses: Theory and Applications, Second Edition, Springer-Verlag, New York (2003).

[2] N. Bacaër, A Short History of Mathematical Population Dynamics, Springer, New York (2011). 
[3] C. Brezinski, Other manifestations of the Schur complement, Linear Algebra Appl., 111 (1988), 231-247.

[4] J.C. Butcher, P. Chartier, The effective order of singly-implicit Runge-Kutta methods, Numerical Algorithms, 20 (1999), 269-284.

[5] R. Penrose, On best approximate solutions of linear matrix equations, Proc. Cambridge Philos. Soc., 52 (1955), 17-19.

[6] D. Poole, Linear Algebra: A Modern Introduction, 2nd Ed., Thomson Learning, London (2006).

[7] W. Wanicharpichat, Explicit minimum polynomial, eigenvector and inverse formula of doubly Leslie Matrix, J. Appl. Math. \& Informatics, 33, No-s: 3-4 (2015), 247-260. 